\title{
MINORÍAS MIGRANTES EN EUROPA: LA EXPULSIÓN DE GITANOS EN ITALIA Y FRANCIA (2008 Y 2010) EN LA PRENSA ESPAÑOLA
}

\author{
Migrant minorities in Europe: The expulsion of \\ Romani people by Italy and France (2008 and 2010) in \\ the Spanish newspapers
}

\section{Joan M. Oleaque-Moreno}

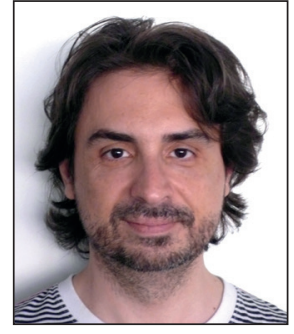

\section{Resumen}

Se examina el tratamiento de los gitanos en la prensa de referencia española durante su expulsión de Italia y Francia en 2008 y 2010, durante los gobiernos de Sarkozy y Berlusconi, por ser España vista como un potencial territorio receptor de esta minoría migrante. Para la realización del estudio, se analiza la cobertura de los diarios El país, ABC y La vanguardia. El interés del caso radica en que es considerado un precedente de las tensiones que ha generado la llegada de refugiados en los últimos años. Los métodos utilizados son el análisis cuantitativo del corpus lingüístico en 474 textos y un análisis crítico del discurso en una selección de los mismos. Los resultados muestran una asociación de la minoría migrante con polémica y marginación, y que el tratamiento se aleja de las pautas trazadas por los libros de estilo para la cobertura de minorías en los medios analizados.

\section{Palabras clave}

Periodismo; Diarios; Periodismo político; Minorías étnicas; Gitanos; Estereotipos; Ética; Libros de estilo; Análisis crítico del discurso.

\section{Abstract}

This paper aims to examine the treatment of gypsies in the Spanish press during their expulsion from Italy and France in 2008 and 2010, under the governments of Sarkozy and Berlusconi, because Spain was considered a potential receiving territory of this migrant minority. For the study, the newspapers El país, ABC, and La vanguardia were selected. The interest in this case is that it can be considered an antecedent to the tensions that the arrival of refugees in recent years have generated. The methods used were the quantitative analysis of the linguistic corpus in 474 texts and a critical analysis of the discourse in a selection of the sample. The results show an association of the migrant minority with controversy and marginalization, and that the treatment moves away from the guidelines drawn by the style books of the newspapers taken into consideration.

\section{Keywords}

Journalism; Newspapers; Political journalism; Ethnic minorities; Gypsies; Stereotypes; Ethics; Style books; Critical discourse analysis.

Oleaque-Moreno, Joan M. (2017). "Minorías migrantes en Europa: la expulsión de gitanos en Italia y Francia (2008 y 2010) en la prensa española”. El profesional de la información, v. 26, n. 2, pp. 211-217.

\section{https://doi.org/10.3145/epi.2017.mar.07}




\section{Introducción}

El presente artículo aborda cómo cubre la prensa española de referencia la persecución y expulsión de los gitanos en 2008 en Italia y en 2010 en Francia. Este hecho se considera un precedente de la situación en 2015 y 2016 en la Unión Europea por la llegada de un millón de refugiados de Siria, Afganistán y otros países (Arango et al., 2016).

La minoría gitana, que alcanza en el continente europeo los diez millones de personas, mantiene presencia en Europa desde el siglo XIV, y en España desde el XV (Aguirre-Felipe, 2006). Los estudios sobre la representación periodística de los gitanos como grupo tienen una trayectoria escasa en la investigación académica y los que se han llevado a cabo han puesto de manifiesto la existencia de un vacío significativo. Contribuir a reducir este vacío en la investigación es un objetivo general de este trabajo.

Como objetivo particular, nos centramos en la cobertura brindada desde la prensa española de referencia, dado que España se presenta en ese momento como país receptor (Piñols, 2010; Reyes, 2010), para identificar claves o elementos presentes en el discurso mediático analizado que ayuden a reconstruir los antecedentes de discusiones posteriores, como las de la campaña sobre la salida del Reino Unido de la Unión Europea y la campaña presidencial en los Estados Unidos (Roberts, 2016).

\section{Estado de la cuestión}

Sobre los mecanismos de transmisión de la discriminación, Van-Dijk (2009) puso de manifiesto cómo los diarios en España se acercan a la minoría gitana en general, en el marco de estudios más complejos que correlacionan el racismo y los medios de comunicación. Bajo su punto de vista, los principales periódicos representarían líneas editoriales e ideas bastante paralelas a las de los partidos políticos mayoritarios. Concretamente, respecto de los titulares periodísticos, plantea que tienden a enfatizar las características negativas de la minoría étnica gitana y las diferencias culturales.

El trabajo de Del-Río-Pedraza (2011), que analiza dos periódicos de tirada nacional (El país y El mundo) y dos andaluces (Diario de Jerez e Información Jerez) entre los años 2005 y 2010, destaca que el léxico utilizado es peyorativo, que el tema sitúa a los gitanos en marcos muy marginales y que las estructuras sintácticas indican, o bien pasividad, o bien una carga negativa en aquellas acciones en las que los gitanos son sujetos activos.

En otros trabajos posteriores se observan representaciones similares. Como indica en su revisión de esta cuestión Tortajada (2009), en el caso de los gitanos inmigrantes se intensifican las características negativas mediante aspectos muy concretos como la mujer con niños que pide en la calle, o niños y niñas sin escolarizar.

Richardson (2012) documenta los métodos de persecución y de discriminación hacia los gitanos y sus cambios de territorio en el Reino Unido, a través del análisis de la prensa. Para esta autora, la delimitación legal y política respecto a dónde pueden establecerse los gitanos, por mantener un modo de vida itinerante, es un mecanismo de control por parte de la sociedad mayoritaria. Este mecanismo se refuerza por la visión mediática, que apunta al colectivo como generador de problemas para los ciudadanos sedentarios.

En Rumanía, de donde proviene la mayoría de migraciones hacia Europa occidental, Mădroane (2012) concluye que la configuración discursiva de las identidades colectivas en la prensa otorga preeminencia a una concepción nacional rumana de la que los gitanos no formarían parte. Y que de hecho los diarios agradecían -aunque con eufemismos- que tuviera lugar la emigración gitana hacia otros países.

En el análisis que López-Catalán y Aharchi (2012) realizan sobre el tratamiento periodístico de los gitanos rumanos inmigrados a Barcelona en La vanguardia, El periódico de Catalunya y la edición catalana de El país, entre 2006 y 2011, se centran en una serie de conflictos vecinales en Badalona. De acuerdo con los resultados de su investigación, los temas con los que se etiquetan las informaciones y las formas de presentar a la población gitana se asocian generalmente a categorías negativas. Se mantiene un discurso asociado al término "invasión" para calificar a los inmigrantes y se obvia que estos colectivos de gitanos llegados del Este, ocupan en la ciudad catalana zonas tradicionalmente de exclusión; la llegada de estos nuevos habitantes lleva a una nueva marginalidad aún más compleja si cabe.

La persecución de gitanos en Italia y Francia es un precedente de la política migratoria que se da en la Unión Europea por la llegada de un millón de refugiados

Los estudios realizados muestran cómo los medios asocian a la minoría gitana, a veces en procesos de migración, con problemas sociales y desórdenes urbanísticos. El repertorio de justificaciones empleadas desde el punto de vista discriminatorio se basa en las características sociales, diferencias culturales y supuestas incompatibilidades grupales, aplicables a la minoría étnica, que además se refuerzan si viene de fuera. Se activa así una efectiva deslegitimación del otro más contemporánea, que se adecúa a las crisis migratorias y tiempos actuales (Wodak, 2015).

\section{Objetivos e hipótesis}

Esta investigación tiene como objetivo principal identificar la representación de los gitanos como grupo en la prensa española de referencia durante los años 2008 y 2010 cuando España es un posible país receptor de los romaníes expulsados de Italia y Francia. El estudio tiene además tres objetivos particulares:

- analizar el corpus textual publicado sobre gitanos durante ambos años para identificar los términos vinculados al grupo objeto de investigación;

- detectar las estrategias discursivas de los medios que van a analizarse en la representación de los gitanos;

- examinar el cumplimiento de lo que esos medios indiquen en sus propios libros de estilo sobre el trato a minorías étnicas.

Como primera hipótesis de la investigación, de acuerdo con 
los estudios previos realizados, se espera encontrar una insistencia periodística en estereotipos étnicos que, por el contexto concreto de prevención frente a nuevas migraciones de los expulsados, provocarán un tratamiento negativo de la comunidad gitana.

Como segunda hipótesis se plantea la existencia de dos discursos:

- una auto-presentación positiva del grupo de "nosotros", formado por quienes escriben y/o editan los textos y por a quienes va potencialmente dirigido (la sociedad mayoritaria);

- una presentación negativa del grupo de "ellos", que en este estudio la componen los gitanos como grupo.

En tercer lugar se espera encontrar una falta de correspondencia entre el tratamiento mediático y las recomendaciones que plantean los libros de estilo de los medios seleccionados para el análisis, en la representación de los gitanos como minoría.

\section{Metodología}

Para dar respuesta a los objetivos planteados se optó por una combinación de métodos cuantitativos y cualitativos. Los medios escogidos para la investigación son $E /$ país, $A B C$ y La vanguardia, diarios con líneas editoriales diferenciadas, gran difusión, superior a 200.000 ejemplares en todos los casos, e influencia socio-política.

Se llevó a cabo en primer lugar un análisis cuantitativo utilizando el software AntConc, que mide las frecuencias de la utilización de palabras en el lenguaje en uso, entre otras funciones.

Se trabajó con su herramienta Collocates, que ordena las colocaciones de términos, es decir, las palabras en combinación que aparecen más repetidamente al lado de un núcleo léxico determinado. En nuestro estudio ese núcleo será gitan (el propio de las palabras gitano/a/os/as) y se utilizó un arco habitual de búsqueda que comprende cinco palabras por su izquierda y hasta cinco palabras por su derecha (Gabrielatos; Baker, 2008). Para una mejor comprensión de la lista de términos seleccionada, se eliminaron los que no tenían significado para la investigación (como artículos definidos e indefinidos, numerales o palabras muy generales). Se tienen en cuenta para el análisis del corpus lingüístico todos aquellos textos periodísticos cuya extensión supere la del breve entre los que contengan la palabra gitano, gitana, gitanos o gitanas publicados en los tres medios, en 2008 y en 2010.

En total se analizaron 474 piezas:

- 83 textos en 2008 y 109 en 2010 en El país;

- 86 en 2008 y 80 en 2010 en $A B C$; y

- 35 en 2008 y 81 en 2010 en La vanguardia.

En segundo lugar se seleccionó un subconjunto de textos que se sometieron a un análisis crítico del discurso (Baker et al., 2008). El análisis crítico del discurso está asociado en particular con la escuela filosófica de Frankfurt (Fairclough; Wodak, 2002) y busca la revisión profunda de textos en su contexto social, con un claro interés por detectar relaciones de discriminación y de control de los más desfavorecidos. Entre otros razonamientos, asume que el lenguaje y la transmisión de comunicación llega a ser una forma de poder. Ha dado forma paulatinamente a una postura crítica, una actitud, un movimiento de investigación que busca desvelar la reproducción discursiva de la discriminación, revisando la situación de los grupos sociales marginalizados (Van-Dijk, 2002). Dentro de esta corriente de estudios, se adoptó para la presente investigación el enfoque histórico-discursivo, impulsado por Wodak y sus colaboradores (Reisigl; Wodak, 2015), que analiza la presencia de estrategias discursivas ideológicas y examina los instrumentos y realizaciones del uso del lenguaje en torno a los estereotipos discriminatorios y que toma en consideración la perspectiva histórica y política y su evolución.

\section{El análisis crítico del discurso detecta en los textos una auto-presentación positi- va de "nosotros" y una presentación ne- gativa de "ellos" (los gitanos)}

Este análisis del discurso se aplica a una muestra de seis textos, uno por año de cada medio. Los criterios de selección no discriminan por género periodístico y están basados en su aparición en portada y en su extensión. Es al menos de una página, y tomamos siempre el texto más amplio que se haya dedicado en ese período a la representación de los gitanos, puesto que el enfoque histórico discursivo requiere piezas de entidad especialmente reseñable con un enfoque más reposado para observar las estrategias más reiteradas que resulten representativas.

La selección comprende los siguientes textos:

- "Los gitanos y la materia de los sueños" (El país, 11 junio 2008, p. 37); https://goo.gl/bm2B4C

- "Noches de sangre y fuego en Roquetas" ( $A B C$, 8 septiembre 208, p. 22); https://goo.gl/OgDv1x

- "La política de inmigración de Berlusconi dispara la alarma en el Gobierno español" (La vanguardia, 17 mayo 2008, p. 15);

- "Los escobazos de Sarkozy" (El país, 5 septiembre 2010, p. 2, sección Domingo); https://goo.gl/HuYpjx

-"Gitanos rumanos de ida y vuelta" ( $A B C$, 19 septiembre 2010, p. 38); https://goo.gl/IsGu9M

- "El Gobierno carga contra el PP sin censurar a Sarkozy por los gitanos" (La vanguardia, 18 septiembre 2010, p. 14).

Se comprobó asimismo el cumplimiento de las recomendaciones sobre cobertura de minorías mencionados en los correspondientes libros de estilo de los tres medios escogidos, que contienen referencias explícitas.

El libro de estilo de El país (2002) indica que no deben utilizarse palabras, frases o expresiones que puedan ser ofensivas para una comunidad, además de recordar que etnia no puede usarse como sinónimo de raza -porque puede diferenciar y catalogar a un grupo- y que nunca deben utilizarse sobre personas inmigrantes palabras como ilegal. 


\section{INTERNACIONAL}

\section{Gitanos rumanos de ida y vuelta}

El Gobierno francés alerta a Europa de la instauración de un «turismo de la miseria» procedente del Este

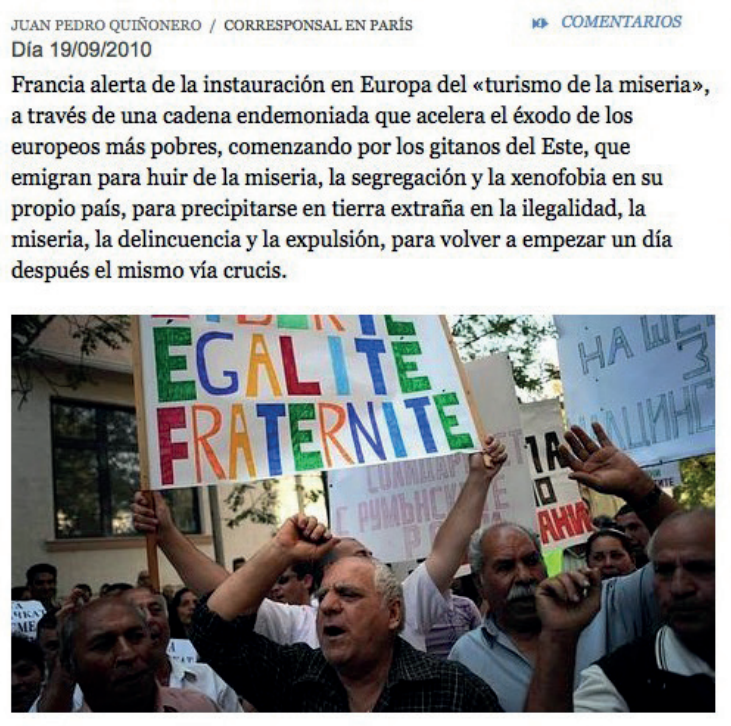

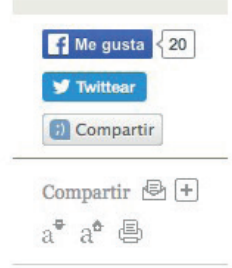

Noticias relacionadas

- Merkel niega haber dicho a Sarkozy que vaya a desmantelar campamentos gitanos

Expertos de $\mathrm{ABC}$ opinan sobre la polémica con los gitanos

Gitanos sin romance, por José María Car

Zapatero carga contra la Comisión Europea por sus acusaciones a polémica sobre los gitanos http://www.abc.es/20100919/internacional/gitanos-rumanos-vuelta-20100919.html
El término "contra" aparece en contextos en que se indica que el gobierno actúa "contra" inmigrantes gitanos o "campamentos", que refuerza una imagen basada en estereotipos y conecta con la representación actual de los "campamentos de refugiados", en otro contexto distinto, así como términos como "disturbios" o "ajuste", que ligan la etnia a la violencia ("ajuste de cuentas"). La referencia a otros colectivos como "senegalés" apunta a una unión con otros grupos inmigrantes.

En 2010 se observa en los tres medios el uso de términos con un significado más unido al conflicto (sin necesidad de que sea provocado por el grupo étnico objeto de estudio), como "expulsión", "problema" o "deportación", que es como la Comisión Europea calificó al conjunto de acciones sobre gitanos en Francia. Aparecen también palabras con significado prácticamente contrario, como "inserción" o "integración”, lo que conecta con la discusión más reciente sobre los colectivos desplazados y el terrorismo internacional, situándonos en una de las raíces del conflicto. En $A B C$, el término "Gallinero" hace referencia a una zona chabolista madrileña donde habita esta población, lo que podría reforzar los estereotipos.
El libro de estilo del grupo Vocento (2003) -al que pertenece $A B C$ - indica que el periodista no discriminará a ninguna fuente proveniente de una u otra minoría social, y que cabe desterrar del lenguaje de sus piezas las expresiones que puedan ser ofensivas para esas mismas minorías.

Por último, La vanguardia (2004) califica como incorrecta la palabra raza en su manual, ya que implica diferencias genéticas, recomendando el uso de etnia, ya que refiere aspectos distintivos culturales entre comunidades. Proscribe también el uso de ilegal en referencia a una persona, e indica la necesidad de evitar la identificación innecesaria de colectivos pertenecientes a una etnia o religión cuando estos datos no sean relevantes para la información.

\section{Resultados}

El estudio cuantitativo del corpus lingüístico muestra coincidencias en los términos que más aparecen junto al núcleo léxico gitan en todos los diarios y en los dos períodos examinados, en la prensa española analizada.

Los términos asociados con mayor frecuencia incluyen referencias a "campamento", "emergencia", "familia" y "disturbios", junto con otros términos identificativos del contexto en 2008 y 2010. aparición
En 2008, las referencias a Italia son habituales en los tres medios ("Berlusconi", "Roma", "Nápoles", "Italia"), y también la insistencia constante en el origen de los gitanos perseguidos en ese país ("rumanos"). En 2010, el origen migrante se mantiene y se completa con referencias a Sarkozy y Francia.

El análisis crítico del discurso en los textos escogidos permite detectar la existencia de una auto-presentación positiva de "nosotros" y una presentación negativa de "ellos" (los gitanos como "el otro"). Los gitanos como grupo son enunciados preeminentemente con colectividades vagas, y apenas

Tabla 1. Términos más utilizados en 2008 por los diarios, asociados a gitano/a/os/as, por frecuencia de

\begin{tabular}{|c|l|c|l|c|l|}
\hline \multicolumn{2}{|c|}{ El país } & \multicolumn{2}{c|}{ ABC* } & \multicolumn{2}{c|}{ La vanguardia } \\
\hline Frecuencia & \multicolumn{1}{|c|}{ Término } & Frecuencia & Término & Frecuencia & \multicolumn{1}{c|}{ Término } \\
\hline 25 & Italia & 6 & rumanos & 10 & Italia \\
\hline 19 & etnia & 4 & Roma & 9 & contra \\
\hline 15 & contra & 4 & etnia & 9 & campamentos \\
\hline 13 & emergencia & 3 & Italia & 8 & rumanos \\
\hline 13 & campamentos & 2 & senegalés & 8 & italianos \\
\hline 12 & rumanos & 2 & población & 7 & viven \\
\hline 11 & población & 2 & payo & 7 & origen \\
\hline 10 & Roma & 2 & joven & 7 & Nápoles \\
\hline 10 & familias & 2 & disturbios & 6 & familias \\
\hline 6 & Berlusconi & 2 & ajuste & 5 & rumano \\
\hline
\end{tabular}

*En el caso de $A B C$, por la conversión del formato pdf a txt en las piezas informativas, el programa ofrece una frecuencia de repetición inferior a la de los otros medios. 
Tabla 2. Términos más utilizados en 2010 por los diarios, asociados a gitano/a/os/as, por frecuencia de aparición

\begin{tabular}{|c|l|c|l|c|l|}
\hline \multicolumn{2}{|c|}{ El país } & \multicolumn{2}{c|}{ ABC } & \multicolumn{2}{c|}{ La vanguardia } \\
\hline Frecuencia & \multicolumn{1}{|c|}{ Término } & Frecuencia & Término & Frecuencia & \multicolumn{1}{c|}{ Término } \\
\hline 86 & rumanos & 26 & rumanos & 54 & rumanos \\
\hline 52 & Francia & 7 & Francia & 21 & expulsión \\
\hline 48 & expulsión & 6 & familia & 16 & Sarkozy \\
\hline 30 & campamentos & 5 & Gallinero & 16 & Francia \\
\hline 22 & Sarkozy & 4 & inmigrantes & 16 & campamentos \\
\hline 20 & etnia & 4 & inserción & 13 & Rumanía \\
\hline 18 & búlgaros & 4 & expulsión & 11 & etnia \\
\hline 16 & deportación & 4 & problema & 7 & ilegales \\
\hline 14 & integración & 3 & Rumanía & 7 & Barcelona \\
\hline 13 & España & 3 & devueltos & 6 & Bruselas \\
\hline
\end{tabular}

se traslada desde los gobiernos de Italia y Francia. $A B C$ sitúa en 2008 la conexión entre etnicidad y migración como parte de una lucha tribal entre "subsaharianos y gitanos" con metáforas bélicas como "noches de sangre y fuego" o "batalla campal". También $A B C$ en 2010 relacionará a los gitanos del Este con una nueva marginalidad transnacional que llama a occidente, usando categorizaciones como "gitanos rumanos de ida y vuelta" o "turismo de la miseria".

En La vanguardia y El país (no en $A B C$ ) se asume que la situación gitana puede deberse a razones de tipo político, pero utilizan perífrasis que evitan aludir a un racismo de

aparecen referidos de manera personalizada. Por ejemplo, "familias gitanas" o "los marginados" o, cuando se habla exclusivamente de gitanos inmigrantes, "los rumanos". El grupo étnico aparece siempre referido como un conjunto ajeno y extraño, que se diferencia por "raza", por "etnia" -como sinónimo de raza- y que se organiza en "clanes", con resonancias tribales.

De los tres medios seleccionados, El país es el único que en 2010 acude a miembros del grupo étnico como fuente de información. Son sin embargo declaraciones poco explícitas, que contrastan mucho con la del grupo de "nosotros", mucho más fluidas y abundantes. Por tanto el predominio casi absoluto es una perspectiva en la que no aparece el punto de vista de la minoría étnica. Tanto en esto como en la referencia a "raza", como a su sustitución indiscriminada por "etnia", los medios contradicen abiertamente sus propios códigos deontológicos.

La representación de "nosotros" busca siempre los elementos respetables de la sociedad mayoritaria, según los entiende cada diario, y según su línea editorial. Por ejemplo, el Gobierno Sarkozy, la Comisión Europea y otras grandes instituciones ( $A B C$, La vanguardia) o la Europa institucional de centro-izquierda (El país).

En general, en los tres diarios la deslegitimación de los gitanos como parte del grupo de "ellos" se produce a través del uso de metáforas y de dichos castizos. Pese a que se suelen referir a los gitanos españoles y a los rumanos como parte de un todo, los gitanos inmigrantes son categorizados siempre como una más oscura versión del grupo étnico, como un colectivo perseguido por naturaleza, "acosado allí donde se asiente" (El país). Se conecta a estos migrantes con el pasado chabolista de los gitanos españoles, y con una especie de destino étnico fatal que se arrastra con la llegada de expulsados ("cada vez más hacia España", titula un despiece en El país).

Hay casos concretos en los que, a través de las estrategias predicativas, se presentan argumentos como acciones "nuestras" que perjudican al grupo de "ellos", conectando a los gitanos con la imagen de elementos sospechosos que carácter histórico. En El país se abunda en la categoría discursiva de la culpa a la víctima, esgrimiendo que "muchos se integran sin dificultad, pero les sigue caracterizando su forma de vivir desarraigada, excluyente y voluntariamente marginada". O que "su carácter apasionado les impulsa a echar mano de la navaja, y a veces corre la sangre".

En La vanguardia se indica que la gitana es "la minoría étnica menos apreciada" de la UE, poniendo el foco sobre "ellos".

En La vanguardia y El país el grupo es descrito a merced de pulsiones, costumbres y determinismos sin escape ("malditos", "incómodos", "patéticos", "poéticos").

Las palabras más repetidas con significado para la investigación suelen ir ligadas a conceptos tribales o ancestrales, como "clan", "raza" o "campamentos"

En $A B C$ se les muestra activos e intervinientes comunitariamente en su propia ruina y dolor ("de mendicidad en familia a la delincuencia sólo había un paso").

En los tres medios la crítica hacia los actos de los políticos sobre los gitanos se presenta cuando la actuación administrativa no concuerda con las líneas editoriales generales del medio. La vanguardia lo ejemplifica especialmente. Así, en 2008 el grupo de "nosotros" se identifica como "España e Italia" y "los cuerpos diplomáticos de ambos países", en un texto que deplora las críticas hechas desde el Gobierno del PSOE del momento a Berlusconi. Sin embargo, en 2010 La vanguardia criticará a ese mismo gobierno socialista, por su ausencia de crítica hacia Sarkozy durante las expulsiones de gitanos. En esos textos el grupo étnico aparece de modo subsidiario.

En cuanto a los libros de estilo, destaca en el análisis del corpus la presencia del término "ilegales" en el diario $L a$ vanguardia, una palabra desaconsejada por su manual de estilo y en algunos casos, como se ha mostrado en las líneas anteriores, se roza la línea de lo que se puede considerar 
como ofensivo para una comunidad, lo que recogen los libros de estilo de El país (2002) y del grupo Vocento (2003).

\section{Conclusiones}

Tras analizar el uso de términos y de estrategias discursivas en tres diarios españoles, respecto de la expulsión de miembros de la minoría gitana en Italia y Francia, podemos afirmar que se confirman las hipótesis planteadas:

- los medios contribuyen con su tratamiento informativo a una deslegitimación de los gitanos como grupo;

- se puede distinguir un discurso sobre "nosotros" y otro sobre "ellos", donde se pone el foco con una visión negativa;

- se confirma el incumplimiento de los libros de estilo respecto del tratamiento de las minorías en los medios analizados.

Los diarios de referencia analizados se muestran, en su representación de los gitanos, más cercanos a los intereses socio-políticos y socio-económicos dominantes que al punto de vista de las voces más necesitadas o con problemas de visibilidad en relación con sus problemas, como es el caso de la de la minoría étnica objeto de análisis, apoyándose en estereotipos y eludiendo la problemática del racismo.

Los gitanos inmigrantes son categorizados siempre como una versión más oscura del grupo étnico, catalogados como un colectivo perseguido por naturaleza

La limitación en tiempo y forma del estudio ha impedido comparar los períodos analizados con la cobertura que estos mismos medios están ofreciendo con la llegada de refugiados. Sin embargo el análisis llevado a cabo hace evidente que el actual miedo al otro ya se dejaba sentir en los medios más importantes frente a la minoría gitana, como prefacio de lo que sucede hoy y que en la representación hay elementos comunes presentes en el discurso como la ilegalidad o los asentamientos.

El aumento de los movimientos migratorios y el contexto socio-político y económico actual hace necesario incrementar los esfuerzos para analizar cómo los medios de comunicación reflejan a los grupos étnicos y migratorios en períodos vulnerables, así como la adecuación y cumplimiento de los libros de estilo, un elemento clave de la autorregulación en los medios cuyas orientaciones resultan en algunos casos excesivamente generales.

\section{Apoyos}

El presente artículo se ha llevado a cabo en el marco del proyecto de investigación Prodis-net. Procesos de construcción del discurso político en internet. Convergencia de partidos, medios y ciudadanos" (FFI2015-67668-R, Ministerio de Economía y Competitividad), dirigido por Beatriz Gallardo, y del que el autor forma parte como investigador.

\section{Bibliografía}

Aguirre-Felipe, Javier (2006). Historia de las itinerancias gitanas. De la India a Andalucía. Zaragoza: Fernando el Católico. ISBN: 9788478208111

Arango, Joaquín; Mahía, Ramón; Moya, David; SánchezMontijano, Elena (dir.) (2016). El año de los refugiados. Anuario Cidob de la inmigración (2015-2016). Barcelona: Ediciones Bellaterra. https://goo.gl/MF7U1i

Baker, Paul; Gabrielatos, Costas; Khosravinik, Majid; Krzyzanowski, Michak; McEnery, Tony; Wodak, Ruth (2008). "A useful methodological synergy? Combining critical discourse analysis and corpus linguistics to examine discourses of refugees and asylum seekers in the U.K. press". Discourse \& society, v. 19, n. 3, pp. 273-305.

https://goo.gl/6sA6Ng https://doi.org/10.1177/0957926508088962

Del-Río-Pedraza, Flora (2011). "La representación de los gitanos en la prensa española". Historia actual online, v. 26, pp. 191-202.

https://dialnet.unirioja.es/servlet/articulo?codigo $=3747221$

El país (2002). Libro de estilo. Madrid: Aguilar ISBN: 978 8403092235

Fairclough, Norman; Wodak, Ruth (2002). "Análisis crítico del discurso". En: Van-Dijk, Teun (coord.). El discurso como interacción social. Estudios sobre el discurso II. Barcelona. Gedisa. ISBN: 9788474327137

Gabrielatos, Costas; Baker, Paul (2008). "Fleeing, sneaking, flooding: A corpus analysis of discursive constructions of refugees and asylum seekers in the U.K. 1996-2005". Journal of English linguistics, v. 36, n. 1, pp. 5-38. 
https://goo.gl/ko8ceM

https://doi.org/10.1177/0075424207311247

La vanguardia (2004). Libro de redacción de La vanguardia. Barcelona: Ariel. ISBN: 9788434482623

López-Catalán, Óscar; Aharchi, Noura (2012). “Discursos sobre la inmigración rrom (gitana) rumana en Barcelona. Estudio de la representación del 'conflicto vecinal' y los trabajos marginales a partir de El país, La vanguardia y El periódico de Catalunya (2006-2011)". Discurso y sociedad, v. 6, n. 3, pp. 543-590.

http://www.dissoc.org/ediciones/v06n03/DS6(3)Lopez\%20 y\%20Aharchi.pdf

Madroane, Irina-Diana (2012). “Roma, Romanian, European: A media framed battle over identity". Critical approaches to discourse analysis across disciplines, v. $5, \mathrm{n}$. 2, 102-119.

http://www.lancaster.ac.uk/fass/journals/cadaad/wpcontent/uploads/2015/01/Volume-5_Madroane.pdf

Piñols, Àngels (2010). "El PP se exhibe por Badalona con una eurodiputada de Sarkozy". El país, 18 septiembre. https://goo.gl/FeJqSz

Reisigl, Martin; Wodak, Ruth (2015). "The discourse historical approach". En: Wodak, Ruth; Meyer, Michael. Methods of critical discourse analysis, $3^{\text {rd }}$ ed. London: Sage. ISBN: 978 1446282410

Reyes, Antonio (2010). "Los gitanos expulsados por Sarkozy desembarcan en Barcelona". Periodista digital, 10 septiembre. https://goo.gl/hiG3w6
Richardson, Joanna (2012). "Stamp on the camps: The social construction of Gypsies and travelers in Media and political". En: Richardson, Joanna; Ryder, Andrew (eds.). Gypsies and travellers: Empowerment and inclusion in British society. Bristol: The Policy Press, pp. 169-185. ISBN: 9781847428943

Roberts, Yvonne (2016). “'Mama, will you be deported?'. Brexit vote triggers eruption of racism". The observer, 2 julio.

https://www.theguardian.com/politics/2016/jul/02/brexittriggers-racism-climate-of-fear

Tortajada, Iolanda (2009). “¿Qué hay de nuestro aquí? Cómo se perciben en los medios algunas minorías residentes en Cataluña". Zer, v. 14, n. 26, pp. 59-80.

http://www.ehu.eus/zer/hemeroteca/pdfs/zer26-03tortajada.pdf

Van-Dijk, Teun A. (2002). "Discurso y racismo". Persona y sociedad, v. 16, n. 3, pp. 191-205.

http://www.discursos.org/oldarticles/Discurso\%20y\%20 racismo.pdf

Van-Dijk, Teun A. (2009). Dominación étnica y racismo discursivo en España y América Latina. Prejuicios e ideologías racistas en Iberoamérica hoy en día. Barcelona: Gedisa. ISBN: 978849784360

Vocento (2003). Libro de estilo. Gijón: Ediciones Trea. ISBN: 9788497040846

Wodak, Ruth (2015). "Discrimination via discourse". En Bonvillain, Nancy (ed.). The Routledge handbook of linguistic anthropology. London: Routledge, pp. 366-384. ISBN: 978 0415834100

\section{Inforảrea}

\section{Ayudamos a tu organización en la transformación digital y el gobierno de la información}

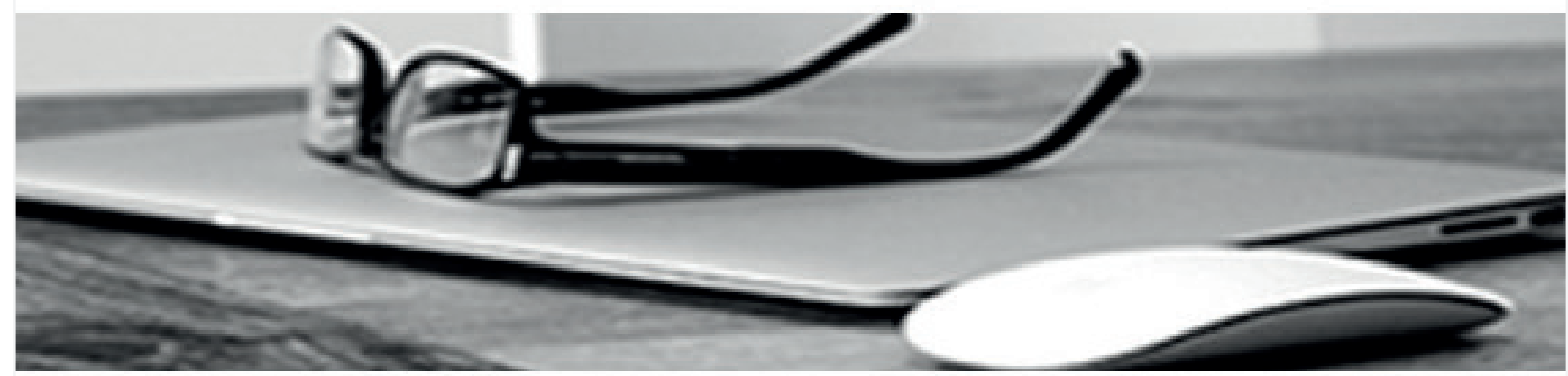

* Consultoría estratégica en gestión y gobierno de la información

* Gestión documental y "records management"

* Gestión de contenidos, intranets corporativas y entornos de colaboración

* Estudios especializados

Clientes satisfechos, cientos de empresas nacionales e internacionales y más de 30 años de experiencia son la mejor garantía de nuestra reputación. 